\title{
Projectile motion via Riemann-Liouville calculus
}

\author{
Bashir Ahmad ${ }^{1 *}$, Hanan Batarfi', Juan J Nieto ${ }^{1,2}$, Óscar Otero-Zarraquiños ${ }^{2}$ and Wafa Shammakh¹
}

${ }^{*}$ Correspondence:

bashirahmad_qau@yahoo.com

${ }^{1}$ Department of Mathematics,

Faculty of Science, King Abdulaziz

University, P.O. Box 80203, Jeddah,

21589, Saudi Arabia

Full list of author information is

available at the end of the article

\begin{abstract}
We present an analysis of projectile motion in view of fractional calculus. We obtain the solution for the problem using the Riemann-Liouville derivative, and then we compute some features of projectile motion in the framework of Riemann-Liouville fractional calculus. We compare the solutions using Caputo derivatives and Riemann-Liouville derivatives.
\end{abstract}

MSC: $70 \mathrm{~B} 05 ; 34 \mathrm{~A} 08$

Keywords: projectile motion; fractional calculus; Riemann-Liouville derivative; Caputo derivative

\section{Analysis of projectile motion in view of Riemann-Liouville fractional calculus}

\subsection{Introduction}

In this paper we consider a projectile motion in view of Riemann-Liouville fractional calculus. The projectile motion is one of the simplest problems whose analogs are ubiquitous in physics. The purpose of this paper is to extend the Caputo approach of [1] to the Riemann-Liouville case. We obtain some new formulas, in particular, the trajectory using the Riemann-Liouville fractional derivative is different. We compare both approaches and indicate new directions of research.

The fractional calculus is an extension of the ordinary calculus and has a history of over 300 years old. It represents a generalization of the ordinary differentiation and integration to arbitrary order and fractional calculus has applications in various fields, e.g. physics, engineering or biology [2-4]. Differential equations of fractional order have assumed a relevant role in the most diverse areas of science and engineering. Some physical considerations in favor of the use of fractional models are given in [5] and fractional mechanics is presented in $[6,7]$.

Many times the authors replace the usual integer derivative by another derivative of fractional order. However, from the physical point of view that is not totally correct [8] and some dimensional correction in the new equation is necessary; for example, substituting a first order derivative $D^{1}:=\frac{d}{d t}$ by $\frac{1}{\sigma^{1-\alpha}} D^{\alpha}$ where $\sigma$ has an appropriate dimension [9].

For some new directions in fractional calculus and fractional differential equations we refer the reader for example to [10-13].

\subsection{Definitions and preliminaries}

We recall some definitions of fractional calculus.

( 2015 Ahmad et al:; licensee Springer. This is an Open Access article distributed under the terms of the Creative Commons Attribution License (http://creativecommons.org/licenses/by/4.0), which permits unrestricted use, distribution, and reproduction in any medium, provided the original work is properly credited. 
The fractional integral of order $\alpha>0$ of a function $f:[0, T] \rightarrow \mathbb{R}$ is defined by

$$
I_{0^{+}}^{\alpha} f(t)=\int_{0}^{t} \frac{(t-s)^{\alpha-1}}{\Gamma(\alpha)} f(s) d s
$$

provided the right-hand side integral exists for almost every $t \in[0, T]$. Here $\Gamma$ is the classical gamma function. This fractional integral is well defined if, for example, $f \in L^{1}(0, T)$.

Let $\alpha>0, n-1<\alpha<n, n \in \mathbb{N}, n \geq 1$.

\subsubsection{Caputo fractional derivative}

Consider the space $A C^{n}[0, T]$ of functions with absolutely continuous derivatives up to order $n-1$ and with absolutely continuous $n$-derivative.

The Caputo fractional derivative of a function $f \in A C^{n}[0, T], T>0$ is defined by [4]

$$
{ }^{c} D^{\alpha} f(t)=\frac{d^{\alpha} f}{d t^{\alpha}}=\frac{1}{\Gamma(n-\alpha)} \int_{0}^{t}(t-\tau)^{n-\alpha-1} f^{(n)}(\tau) d \tau, \quad t \in[0, T] .
$$

The Laplace transform of a function $f:[0, \infty) \rightarrow \mathbb{R}$ is the function $F(s)$,

$$
F(s)=\mathcal{L}\{f(t)\}=\int_{0}^{\infty} e^{-s t} f(t) d t
$$

provided it is well defined.

If we apply the Laplace transform to (1.1) we get [4]

$$
\mathcal{L}\left\{{ }^{c} D^{\alpha} f(t)\right\}=s^{\alpha} F(s)-\sum_{m=0}^{n-1} s^{\alpha-m-1} f^{(m)}(0) .
$$

\subsubsection{Riemann-Liouville fractional derivative}

The Riemann-Liouville fractional derivative of a function $f$ is defined as

$$
D^{\alpha} f(t)=\frac{d^{\alpha} f}{d t^{\alpha}}=\frac{1}{\Gamma(n-\alpha)}\left(\frac{d}{d t}\right)^{n} \int_{0}^{t}(t-\tau)^{-\alpha+n-1} f(\tau) d \tau,
$$

provided the left-hand side is defined for almost every $t>0$. If we apply the Laplace transform, we get

$$
\mathcal{L}\left\{D^{\alpha} f(t)\right\}=s^{\alpha} F(s)-\sum_{m=0}^{n-1} s^{m} f^{(\alpha-m-1)}(0) .
$$

We recall that the Caputo derivative of a constant function is zero, i.e., ${ }^{c} D^{\alpha}(1)=0$. However, for the Riemann-Liouville derivative:

$$
D^{\alpha} 1=\frac{1}{\Gamma(1-\alpha)} t^{-\alpha} .
$$

Also $D^{\alpha} t^{\alpha-j}=0$ for $j=1,2, \ldots,[\alpha]+1$.

A useful formula is the following relation:

$$
D^{\alpha} t^{\gamma}=\frac{\Gamma(\gamma+1)}{\Gamma(\gamma+1-\alpha)} t^{\gamma-\alpha}, \quad \alpha>0, \gamma-1>0, t>0,
$$

and for $\gamma=0$ we obtain the Riemann-Liouville derivative of a constant. 


\subsubsection{Mittag-Leffler function}

A two-parameter function of the Mittag-Leffler type is defined by the series expansion [4]:

$$
E_{\alpha, \beta}(z)=\sum_{m=0}^{\infty} \frac{z^{m}}{\Gamma(\alpha m+\beta)} \quad(\alpha>0, \beta>0) .
$$

The Laplace transform for the Mittag-Leffler function is very useful in solving fractional differential equations:

$$
\int_{0}^{\infty} e^{-s t} t^{\alpha m+\beta-1} E_{\alpha, \beta}^{(m)}\left( \pm a t^{\alpha}\right) d t=\frac{m ! s^{\alpha-\beta}}{\left(s^{\alpha} \pm a\right)^{m+1}} \quad\left(\operatorname{Re}(s)>a^{\frac{1}{\alpha}}\right) .
$$

Hence

$$
\mathcal{L}^{-1}\left[\frac{m ! s^{\alpha-\beta}}{\left(s^{\alpha} \pm a\right)^{m+1}}\right]=t^{\alpha m+\beta-1} E_{\alpha, \beta}^{(m)}\left( \pm a t^{\alpha}\right) .
$$

The general solution of the following simple fractional differential equation:

$$
{ }^{c} D^{\alpha} f=0
$$

is given by

$$
f(t)=c_{0}+c_{1} t+\cdots+c_{n-1} t^{n-1}
$$

with $c_{0}, c_{1}, \ldots, c_{n-1}$ arbitrary constants.

Thus for $0<\alpha<1$, the general solution of ${ }^{c} D^{\alpha} f=0$ is a constant.

However, for the Riemann-Liouville derivative we find that the general solution of

$$
D^{\alpha} f=0
$$

is given by

$$
f(t)=c_{0} t^{\alpha-n}+c_{1} t^{\alpha-n+1}+\cdots+c_{n-1} t^{\alpha-1}
$$

Thus for $0<\alpha<1$, the general solution of

$$
D^{\alpha} f=0 \text { is } f(t)=c t^{\alpha-1}, \quad c \in \mathbb{R} .
$$

This is a crucial difference since $t^{\alpha-1}$ has a singularity at $t=0^{+}$.

\subsection{Classical problem formulation of projectile motion}

A familiar basic physics problem involves the determination of the motion of an object which is projected into a spatial medium and subject to a uniform gravitational field. In this section, we consider the introductory version of this problem in which the medium usually does not offer resistance to the projectile motion. The projectile is treated as a particle of mass $m$ under an uniform gravitational force and no drag force is considered. 
Under these conditions, the classical equations of motion for the particle, in the $x-y$ plane, are given by

$$
m \frac{d^{2} x}{d t^{2}}=0, \quad m \frac{d^{2} y}{d t^{2}}=-m g
$$

by the classic Newton law.

The corresponding initial conditions are

$$
\begin{array}{ll}
x(0)=0, & \dot{x}(0)=v_{0} \cos \phi, \\
y(0)=0, & \dot{y}(0)=v_{0} \sin \phi ;
\end{array}
$$

namely, the projectile starts from rest, with an initial force of module $v_{0}$ and an angle $\phi$.

The trajectory is given by a parabola:

$$
y=\tan \phi x-\frac{g}{2 v_{0}^{2}} \sec ^{2} \phi x^{2} .
$$

The range is the horizontal distance traveled by the projectile from the time it is fired until it lands. The maximum altitude is the height of the highest point in the trajectory. The time of flight is the amount of time the projectile spends in the air between when it is fired and when it lands.

The range is

$$
\frac{2 v_{0}^{2}}{g} \sin \phi \cos \phi
$$

and the corresponding flight time is

$$
\frac{2 v_{0} \sin \phi}{g}
$$

The range is maximum for $\phi=\frac{\pi}{4}$. Finally the maximum height is equal to

$$
\frac{v_{0}^{2} \sin ^{2} \phi}{2 g}
$$

\subsection{Caputo fractional problem formulation}

Now consider the above problem in view of the fractional calculus. Before doing so, we consider the question of how to formulate the acceleration of a particle in the fractional approach. It seems reasonable to consider instead of the second derivative a fractional order derivative [1]. The fractional differential equations for the projectile problem is then

$$
\frac{d^{\alpha} x}{d t^{\alpha}}=0, \quad \frac{d^{\alpha} y}{d t^{\alpha}}=-g
$$

where $1<\alpha \leq 2$.

For $\alpha=2$ we recover, of course, the classical case (1.9).

Physically, we can interpret the fractional derivatives of $x$ and $y$, respectively, as the accelerations of the projectile in the horizontal and vertical directions, which reduce to the acceleration of the classical mechanics at $\alpha \rightarrow 2^{-}$. 
First we recall the solutions using the Caputo derivative [1]. The general solution of (1.11) using the Caputo derivative is

$$
x(t)=c_{1}+c_{2} t, \quad y(t)=-g \frac{t^{\alpha}}{\Gamma(\alpha+1)}+d_{1}+d_{2} t .
$$

Implementing the initial conditions (1.10) we get

$$
x(t)=v_{0} \cos \phi t, \quad y(t)=-g \frac{t^{\alpha}}{\Gamma(\alpha+1)}+v_{0} \sin \phi t .
$$

For the Caputo derivative the solution of (1.11) can also be obtained by means of Laplace transform (1.2) as in [1]:

$$
\begin{aligned}
& s^{\alpha} X(s)-s^{\alpha-1} x(0)-s^{\alpha-2} \dot{x}(0)=0, \\
& s^{\alpha} Y(s)-s^{\alpha-1} y(0)-s^{\alpha-2} \dot{y}(0)=-\frac{g}{s} .
\end{aligned}
$$

Using the initial conditions (1.10), we get

$$
X(s)=\frac{\nu_{0} \cos \phi}{s^{2}}, \quad Y(s)=-\frac{g}{s^{\alpha+1}}+\frac{\nu_{0} \sin \phi}{s^{2}},
$$

and then $x, y$ are given by (1.12).

\subsection{Riemann-Liouville fractional problem formulation}

For the Riemann-Liouville derivative the corresponding and adequate initial conditions are [2]

$$
\begin{array}{ll}
D^{\alpha-2} x(0)=0, & D^{\alpha-1} x(0)=v_{0} \cos \phi, \\
D^{\alpha-2} y(0)=0, & D^{\alpha-1} y(0)=v_{0} \sin \phi,
\end{array}
$$

which, of course, coincide with the initial conditions (1.10) for $\alpha=2$.

The general solution of (1.11) for the Riemann-Liouville derivative is given by

$$
\begin{aligned}
& x(t)=c_{0} t^{\alpha-2}+c_{1} t^{\alpha-1}, \\
& y(t)=d_{0} t^{\alpha-2}+d_{1} t^{\alpha-1}-\frac{g}{\Gamma(\alpha+1)} t^{\alpha},
\end{aligned}
$$

in view of (1.8).

However,

$$
D^{\alpha-1} x(t)=c_{0} D^{\alpha-1}\left(t^{\alpha-2}\right)+c_{1} D^{\alpha-1}\left(t^{\alpha-1}\right) .
$$

We have

$$
D^{\alpha-1}\left(t^{\alpha-2}\right)=0
$$

and

$$
D^{\alpha-1}\left(t^{\alpha-1}\right)=\Gamma(\alpha) .
$$


Therefore $D^{\alpha-1} x(t)=c_{1} \Gamma(\alpha)$.

Using the initial condition $D^{\alpha-1} x(0)=v_{0} \cos \phi$, we can conclude that $c_{1}=\frac{v_{0} \cos \phi}{\Gamma(\alpha)}$, so that

$$
x(t)=c_{0} t^{\alpha-2}+\frac{v_{0} \cos \phi}{\Gamma(\alpha)} t^{\alpha-1} .
$$

Using the other initial condition we obtain with $c_{0}$,

$$
D^{\alpha-2} x(t)=c_{0} \Gamma(\alpha-1)+v_{0} \cos \phi t
$$

since $D^{\alpha-2}\left(t^{\alpha-2}\right)=\Gamma(\alpha-1)$ and $D^{\alpha-2}\left(t^{\alpha-1}\right)=\Gamma(\alpha) t$.

Therefore $D^{\alpha-2} x(0)=0$ implies $c_{0}=0$ and we get

$$
x(t)=\frac{v_{0} \cos \phi}{\Gamma(\alpha)} t^{\alpha-1} .
$$

In the same way

$$
\begin{aligned}
& D^{\alpha-1} y(t)=-g t+d_{1} \Gamma(\alpha), \\
& D^{\alpha-2} y(t)=\frac{-1}{2} g t^{2}+d_{0} \Gamma(\alpha-1)+v_{0} \sin \phi t,
\end{aligned}
$$

where we obtain $d_{1}=\frac{v_{0} \sin \phi}{\Gamma(\alpha)}, d_{0}=0$, i.e.,

$$
y(t)=\frac{-g}{\Gamma(\alpha+1)} t^{\alpha}+\frac{v_{0} \sin \phi}{\Gamma(\alpha)} t^{\alpha-1}
$$

We point out that the solution $x(t), y(t)$ given by (1.16), (1.17) is qualitatively different from the solution (1.12). With this Riemann-Liouville approach we get new fractional trajectories.

For the Riemann-Liouville derivative, the solution of (1.11) can also be reached by means of the Laplace transform (1.4) as follows:

$$
\begin{aligned}
& s^{\alpha} X(s)-D^{\alpha-1} x(0)-s D^{\alpha-2} x(0)=0, \\
& s^{\alpha} Y(s)-D^{\alpha-1} y(0)-s D^{\alpha-2} y(0)=-\frac{g}{s} .
\end{aligned}
$$

Using the initial conditions (1.15), we get

$$
X(s)=\frac{\nu_{0} \cos \phi}{s^{\alpha}}, \quad Y(s)=-\frac{g}{s^{\alpha+1}}+\frac{\nu_{0} \sin \phi}{s^{\alpha}} .
$$

From this it follows that $x(t), y(t)$ are given by (1.16), (1.17), respectively.

\subsection{Features of projectile motion in the fractional calculus}

As we have recalled before, three quantities are particularly relevant for identifying, distinguishing, and analyzing trajectories in our setting: the range, the maximum altitude, and the time of flight.

We recall each of these quantities using the Caputo derivative [1] and compute them for the Riemann-Liouville derivative. 


\subsubsection{Trajectory}

Caputo: By eliminating $t$ from (1.12), we obtain the trajectory of the fractional projectile for arbitrary $\alpha$ as:

$$
y=\tan \phi x-\frac{g x^{\alpha}}{\Gamma(\alpha+1)\left(v_{0} \cos \phi\right)^{\alpha}} .
$$

This was obtained in [1].

As $\alpha \rightarrow 2^{-}$, (1.20) gives the classical trajectory equation:

$$
y=\tan \phi x-\frac{g}{2 v_{0}^{2}} \sec ^{2} \phi x^{2}
$$

Riemann-Liouville: By eliminating $t$ from (1.16), (1.17) we now obtain the trajectory of the fractional projectile from (1.16), (1.17):

$$
y=\tan \phi x-\frac{g}{\Gamma(\alpha+1)}\left(\frac{x \Gamma(\alpha)}{v_{0} \cos \phi}\right)^{\frac{\alpha}{\alpha-1}}
$$

As a new result, as $\alpha \rightarrow 2^{-}$, from (1.22) we obtain the classical trajectory equation (1.21).

Observe that (1.22) is different from (1.20).

\subsubsection{Range}

The fractional projectile range is defined as the value of $x$ at the impact point.

Caputo [1]: Thus, $y=0$ at $x=R_{\mathrm{F}}$. Hence, $R_{\mathrm{F}}$ is given as

$$
R_{\mathrm{F}}=\left(v_{0}\right)^{\frac{\alpha}{\alpha-1}}\left[\frac{\Gamma(\alpha+1)}{g}\right]^{\frac{1}{\alpha-1}}(\sin \phi)^{\frac{1}{\alpha-1}} \cos \phi
$$

Also as $\alpha \rightarrow 2^{-},(1.23)$ leads to the range of the classical projectile:

$$
R_{\mathrm{C}}=\frac{2 v_{0}^{2}}{g} \sin \phi \cos \phi
$$

See Figure 1 for $v_{0}=2$.

Riemann-Liouville: Doing again $y=0$ at $x=R_{\mathrm{F}}$, we obtain in this case for $R_{\mathrm{F}}$,

$$
R_{\mathrm{F}}=\frac{v_{0} \cos \phi}{\Gamma(\alpha)}\left(\frac{\alpha v_{0} \sin \phi}{g}\right)^{\alpha-1}
$$

which is different and, surprisingly, simpler than (1.23). See Figure 2.

As $\alpha \rightarrow 2^{-}$, from (1.25) we obtain the range of the classical projectile (1.24).

We compare both ranges in Figure 3.

\subsubsection{Flight time}

The fractional time of flight $t_{\mathrm{F}-\text { flight }}$ is defined as the value of $t$ at which the projectile hits the ground.

Caputo: Thus, $y=0$ at $t=t_{\mathrm{F}-\text { flight }}$, hence

$$
t_{\mathrm{F}-\text { flight }}=\left[\frac{\Gamma(\alpha+1) v_{0} \sin \phi}{g}\right]^{\frac{1}{\alpha-1}} \text {. }
$$




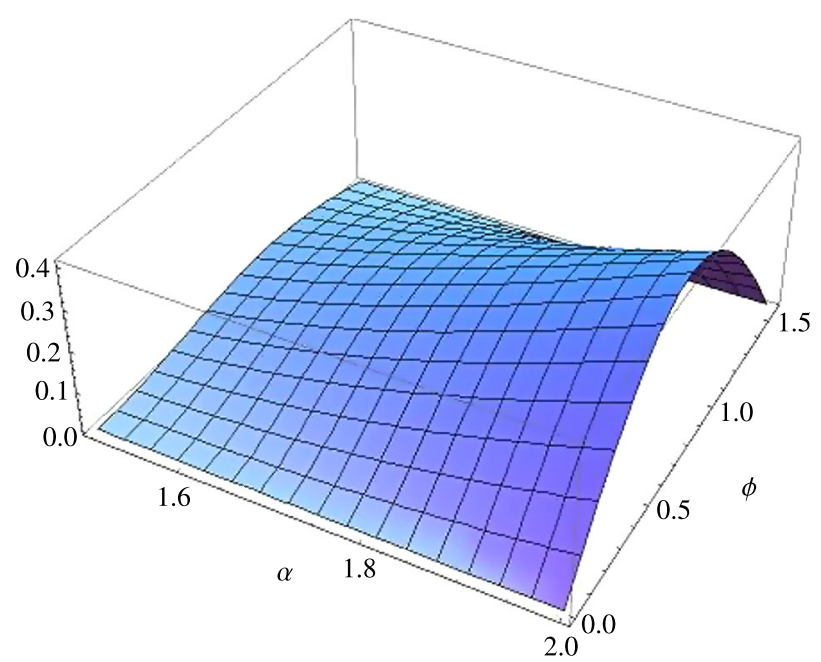

Figure 1 Range (Caputo) depending on the order of differentiation $\alpha, 1<\alpha<2$, and the angle $\phi$, $0 \leq \phi \leq \frac{\pi}{2}\left(v_{0}=2\right)$.

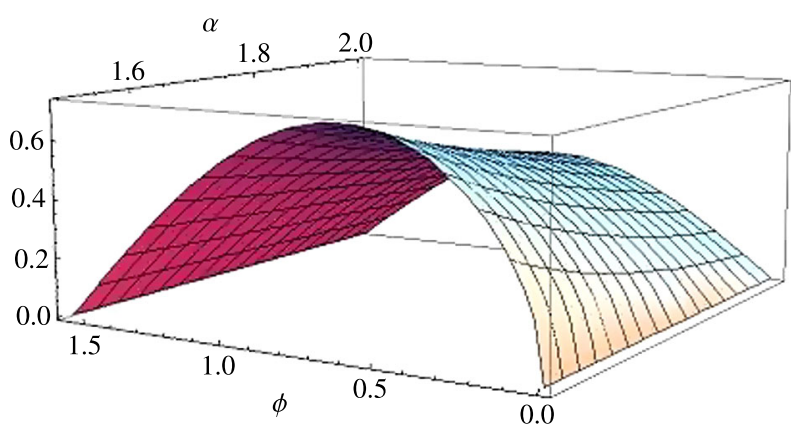

Figure 2 Range (Riemann-Liouville) depending on the order of differentiation $\alpha$ and the angle $\phi$ $\left(v_{0}=2\right)$.

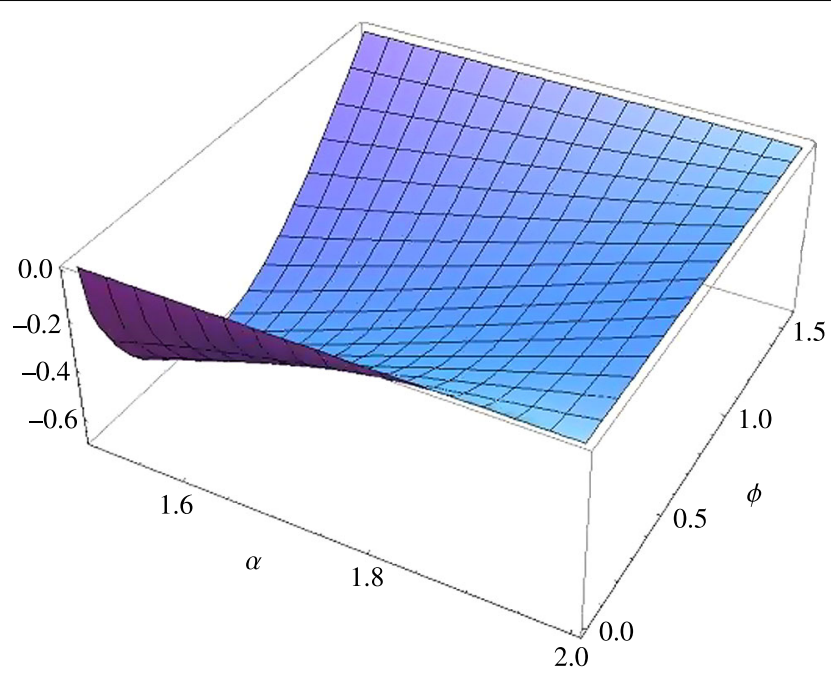

Figure 3 Comparison between ranges depending on the order of differentiation $\alpha$ and the angle $\phi$ $\left(v_{0}=2\right)$. 
Here, it should be noted also that the classical flight time $t_{\mathrm{C}-\text { flight }}$ can be deduced from (1.26) at $\alpha \rightarrow 2^{-}$as

$$
t_{\mathrm{C}-\text { flight }}=\frac{2 v_{0} \sin \phi}{g} .
$$

Riemann-Liouville: Doing $y=0$ at $t=t_{\mathrm{F} \text {-flight }}$, we obtain

$$
t_{\mathrm{F}-\text { flight }}=\frac{\alpha v_{0} \sin \phi}{g} .
$$

This has a simpler expression than the Caputo analogous (1.26).

Again, it is verified that the classical flight time $t_{\mathrm{C} \text {-flight }}$ can be obtained from (1.28) at $\alpha \rightarrow 2^{-}$to get (1.27).

\subsubsection{Maximum height}

Caputo: The projectile reaches its maximum height when its vertical component vanishes, i.e., $\dot{y}=0$. By solving this equation for $t$, we get

$$
t_{\text {height-max }}=\left[\frac{\Gamma(\alpha) v_{0} \sin \phi}{g}\right]^{\frac{1}{\alpha-1}} .
$$

Substituting (1.29) into $y(t)$ in (1.12), we obtain the Caputo fractional maximum height $H_{\mathrm{F}}$ :

$$
H_{\mathrm{F}}=\left(1-\frac{1}{\alpha}\right)\left[\frac{\Gamma(\alpha)}{g}\right]^{\frac{1}{\alpha-1}}\left(v_{0} \sin \phi\right)^{\frac{\alpha}{\alpha-1}} .
$$

Here, we can also obtain the maximum height of the classical projectile when $\alpha \rightarrow 2^{-}$:

$$
H_{\mathrm{C}}=\frac{v_{0}^{2} \sin ^{2} \phi}{2 g} .
$$

Riemann-Liouville: Solving for $t$ the equation $\dot{y}=0$, we obtain

$$
t_{\text {height-max }}=\frac{(\alpha-1) v_{0} \sin \phi}{g} .
$$

Substituting (1.32) into $y(t)$ in (1.17), we obtain the Riemann-Liouville fractional maximum height $H_{\mathrm{F}}$ :

$$
H_{\mathrm{F}}=\frac{-\left((\alpha-1) v_{0} \sin \phi\right)^{\alpha}}{\Gamma(\alpha+1) g^{\alpha-1}}+\frac{\left(v_{0} \sin \phi\right)^{\alpha}}{\Gamma(\alpha)}\left(\frac{\alpha-1}{g}\right)^{\alpha-1} .
$$

If $\alpha \rightarrow 2^{-}$, we obtain again (1.31). We have

$$
H_{\mathrm{C}}=\frac{v_{0}^{2} \sin ^{2} \phi}{2 g} \text {. }
$$

\section{Relationship between the ranges}

In this section we present two main results: one of them comparing the range of a projectile using the fractional calculus by the Caputo derivative with the range in the classical case 
(Theorem 1 of [1]), and the other comparing again the classical range with the fractional range, but using this time the new formulas obtained for the Riemann-Liouville derivative.

Theorem 2.1 ([1]) Suppose that

$$
\mu=\frac{1}{2}[\Gamma(\alpha+1)]^{\frac{1}{\alpha-1}}\left[\frac{v_{0} \sin \phi}{g}\right]^{\frac{2-\alpha}{\alpha-1}},
$$

then the relation between the range of a projectile using fractional calculus (by Caputo) $R_{\mathrm{F}-\mathrm{C}}$ and the range in the classical case $R_{\mathrm{C}}$ is given by $R_{\mathrm{F}-\mathrm{C}}=\mu R_{\mathrm{C}}$, hence:

1. $R_{\mathrm{F}-\mathrm{C}}=R_{\mathrm{C}}$ if $\mu=1$, i.e., $v_{0} \sin \phi=\left[\frac{2^{\alpha-1}}{\Gamma(\alpha+1)}\right] \frac{1}{2-\alpha} g$.

2. $R_{\mathrm{F}-\mathrm{C}}>R_{\mathrm{C}}$ if $\mu>1$, i.e., $v_{0} \sin \phi>\left[\frac{2^{\alpha-1}}{\Gamma(\alpha+1)}\right] \frac{1}{2-\alpha} g$.

3. $R_{\mathrm{F}-\mathrm{C}}<R_{\mathrm{C}}$ if $\mu<1$, i.e., $v_{0} \sin \phi<\left[\frac{2^{\alpha-1}}{\Gamma(\alpha+1)}\right] \frac{1}{2-\alpha} g$.

We now give the corresponding result for the Riemann-Liouville range without proof, since it is similar to that of Theorem 2.1.

Theorem 2.2 Suppose that $v=\frac{g^{2-\alpha}\left(v_{0} \sin \phi\right)^{\alpha-2} \alpha^{\alpha-1}}{2 \Gamma(\alpha)}$, then the relation between the range of a projectile using fractional calculus (by Riemann-Liouville) $R_{\mathrm{F}-\mathrm{RL}}$ and the range in the classical case $R_{\mathrm{C}}$ is given by $R_{\mathrm{F}-\mathrm{RL}}=v R_{\mathrm{C}}$, hence:

1. $R_{\mathrm{F}-\mathrm{RL}}=R_{\mathrm{C}}$ if $v=1$, i.e., $v_{0} \sin \phi=\left(\frac{2 \Gamma(\alpha)}{\alpha^{\alpha-1}}\right)^{\frac{1}{\alpha-2}} \frac{1}{g}$.

2. $R_{\mathrm{F}-\mathrm{RL}}>R_{\mathrm{C}}$ if $v>1$, i.e., $v_{0} \sin \phi>\left(\frac{2 \Gamma(\alpha)}{\alpha^{\alpha-1}}\right)^{\frac{1}{\alpha-2}} \frac{1}{g}$.

3. $R_{\mathrm{F}-\mathrm{RL}}<R_{\mathrm{C}}$ if $v<1$, i.e., $v_{0} \sin \phi<\left(\frac{2 \Gamma(\alpha)}{\alpha^{\alpha-1}}\right)^{\frac{1}{\alpha-2}} \frac{1}{g}$.

\section{Maximum projectile range}

In applications, the maximum projectile range and the required optimal projection angle are of considerable interest (e.g. in situations for which the projectile serves as a delivery system $[1,14,15])$. In order to maximize $R_{\mathrm{F}}$, it is necessary to optimize the projection angle $\phi$. This is developed below.

Theorem 3.1 (see [1], Theorem 3) The optimal projection angle $\phi_{\max }$ and the maximum projectile range $R_{\mathrm{F}-\max }$ (by Caputo) are given by

$$
\phi_{\max }=\tan ^{-1}\left(\frac{1}{\sqrt{\alpha-1}}\right) ; \quad \frac{\pi}{4} \leq \phi_{\max }<\frac{\pi}{2}
$$

and

$$
R_{\mathrm{F}-\max }=\sqrt{\frac{\alpha-1}{\alpha}}\left[\frac{\Gamma(\alpha+1) v_{0}^{\alpha}}{\sqrt{\alpha} g}\right]^{\frac{1}{\alpha-1}} .
$$

See Figure 4 for the optimal angle. For $\alpha=2$ the optimal angle is $\frac{\pi}{4}=0.785398 \ldots$ and the maximum range in Figure 5 for $v_{0}=2$ where for $\alpha=2$, we obtain according to (1.24), an approximate value of 0.408163 .

We now present a new result on the optimal angle with the Riemann-Liouville approach.

Theorem 3.2 The optimal projection angle $\phi_{\max }$ to attain the maximum projectile range $R_{\mathrm{F}-\max }$ (by Riemann-Liouville) is given by

$$
\phi_{\max }=\tan ^{-1}(\sqrt{\alpha-1}) ; \quad \frac{\pi}{4} \leq \phi_{\max }<\frac{\pi}{2},
$$




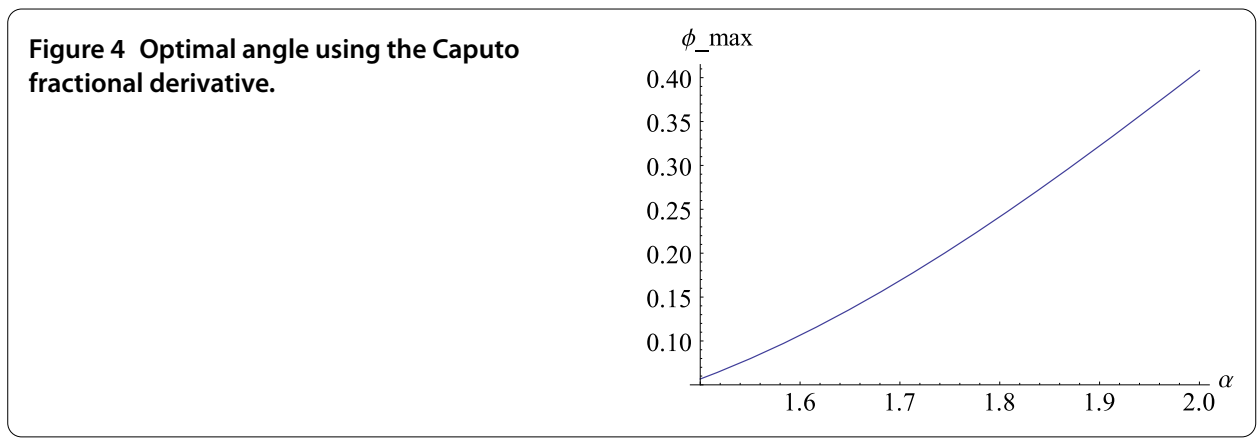

Figure 5 Maximum range using the Caputo fractional derivative $\left(v_{0}=2\right)$.

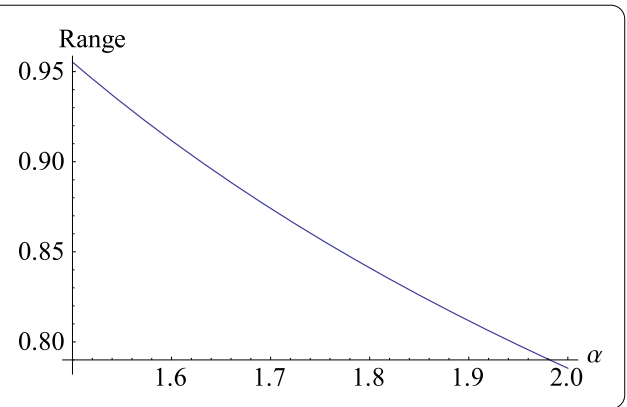

and the corresponding maximum range

$$
R_{\mathrm{F}-\max }=\left(\frac{v_{0}}{\Gamma(\alpha)}\right)^{\alpha}\left(\frac{\Gamma(\alpha+1)}{g}\right)^{\alpha-1} \frac{1}{\sqrt{\alpha}}\left(\frac{\alpha-1}{\alpha}\right)^{\frac{\alpha-1}{2}} .
$$

Proof We have to maximize the function $R_{\mathrm{F}}$ given in (1.25), and for this a necessary condition is $\frac{d R_{\mathrm{F}}}{d \phi}=0$. Therefore,

$$
\frac{d R_{\mathrm{F}}}{d \phi}=\left(\frac{v_{0}}{\Gamma(\alpha)}\right)^{\alpha}\left(\frac{\Gamma(\alpha+1)}{g}\right)^{\alpha-1}\left[(\alpha-1)(\sin \phi)^{\alpha-2}(\cos \phi)^{2}-(\sin \phi)^{\alpha}\right]
$$

The next step is to solve the equation $\frac{d R_{\mathrm{F}}}{d \phi}=0$ as $\phi=\phi_{\max }$, and after some simple calculations we get

$$
\phi_{\max }=\tan ^{-1}(\sqrt{\alpha-1})
$$

On the other hand, $1<\alpha \leq 2^{-}$implies that $1 \leq \frac{1}{\sqrt{\alpha-1}}<\infty$, i.e., $\frac{\pi}{4} \leq \phi_{\max }<\frac{\pi}{2}$.

When in this equation we let $\alpha \rightarrow 2^{-}$, we obtain $\phi_{\max }=\frac{\pi}{4}$, which is the optimal projection angle in the classical case.

Now we need to find the value of the maximum projectile range $R_{\mathrm{F}-\mathrm{max}}$, and for this we replace the $\phi_{\max }$ that we just calculated in (1.25). Using the trigonometrical relations

$$
\sin \phi=\frac{\tan \phi}{\sqrt{1+(\tan \phi)^{2}}}, \quad \cos \phi=\frac{1}{\sqrt{1+(\tan \phi)^{2}}}
$$

simplifies greatly the calculations. 


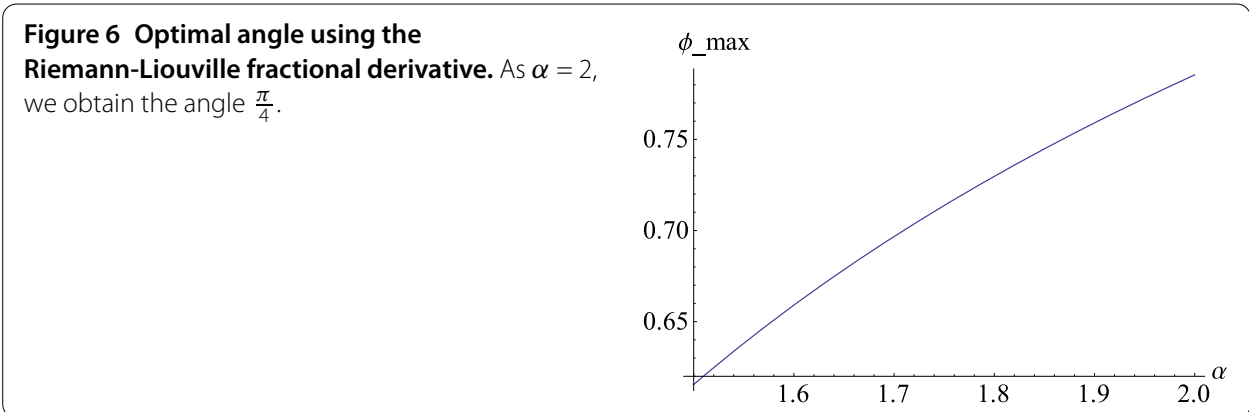

Figure 7 Maximum range using the Riemann-Liouville fractional derivative $\left(v_{0}=2\right)$. As $\alpha=2$, we obtain the value $\frac{8}{9.8} \sin \left(\frac{\pi}{4}\right) \cos \left(\frac{\pi}{4}\right)$.

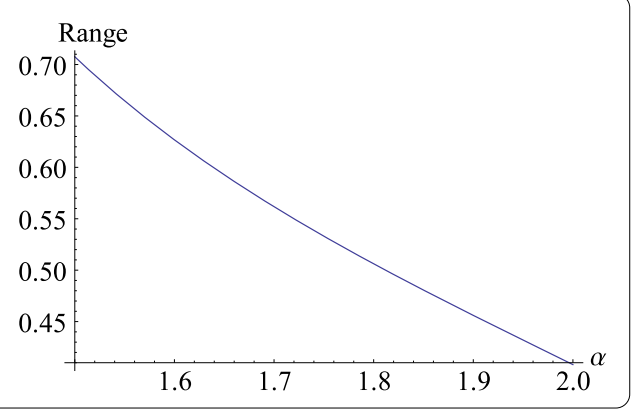

Figure 8 Comparison of the optimal angles.

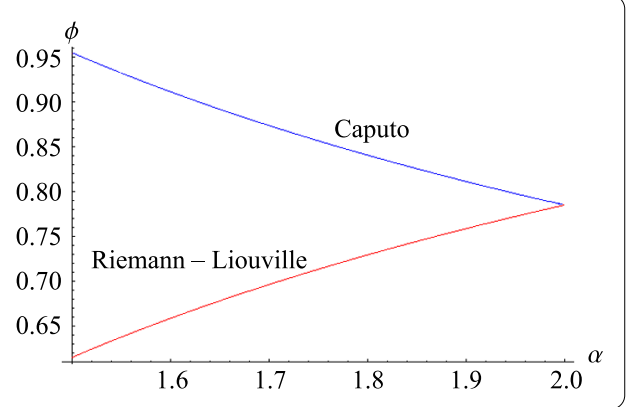

Hence, the formula for the maximum projectile range is given by

$$
R_{\mathrm{F}-\max }=\left(\frac{v_{0}}{\Gamma(\alpha)}\right)^{\alpha}\left(\frac{\Gamma(\alpha+1)}{g}\right)^{\alpha-1} \frac{1}{\sqrt{\alpha}}\left(\frac{\alpha-1}{\alpha}\right)^{\frac{\alpha-1}{2}}
$$

We get from it the expression of the classical maximum range $R_{\mathrm{C}-\max }=\frac{v_{0}^{2}}{g}$ as $\alpha \rightarrow 2^{-}$.

In Figure 6 we show the optimal angle (3.1). We point out a qualitative difference with the optimal angle for the Caputo case: $\phi_{\max }$ is increasing in $\alpha$ when using the RiemannLiouville derivative. Again for $\alpha=2$ we recover the optimal angle $\frac{\pi}{4}$. The maximum range (3.2) is decreasing in $\alpha$ as is shown in Figure 7 for $v_{0}=2$.

Finally, in Figures 8 and 9 we compare the optimal angle and the maximum range for the Caputo and Riemann-Liouville cases, respectively.

\section{Conclusions}

We have studied the motion of a projectile using the Riemann-Liouville fractional derivative. We have compared the trajectory, range, flight time, maximum height, maximum 


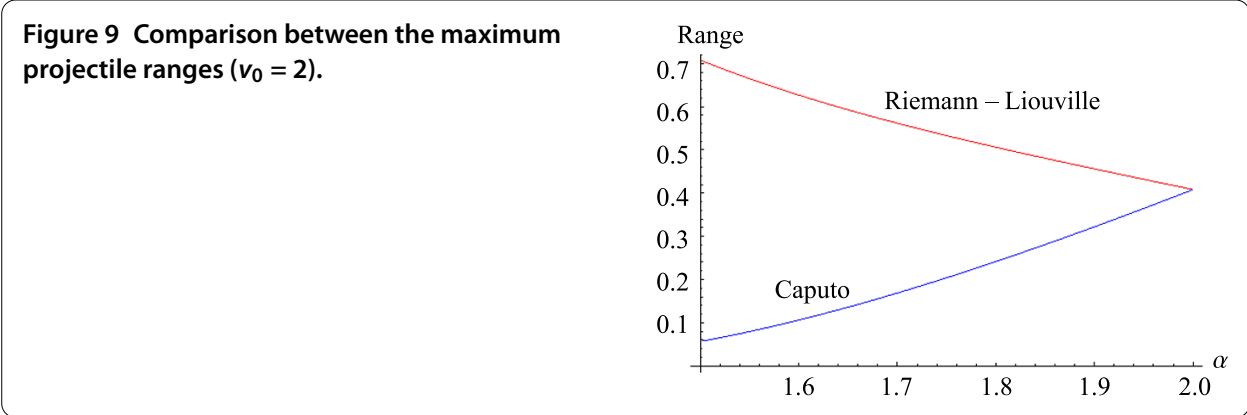

projectile range, and optimal angle with the results obtained previously for the fractional Caputo derivative.

Some relevant qualitative differences between the Caputo and the Riemann-Liouville approach are indicated. For example, the maximum projectile range is increasing with the order of derivative for the Caputo approach and, by contrast, it is decreasing with the order of the derivative for our Riemann-Liouville approach.

In the future we suggest to study the motion of a projectile in a resistant medium via the fractional calculus approach.

\section{Competing interests}

The authors declare that they have no competing interests.

\section{Authors' contributions}

Each of the authors contributed to each part of this study equally and read and approved the final version of the manuscript.

\section{Author details}

'Department of Mathematics, Faculty of Science, King Abdulaziz University, P.O. Box 80203, Jeddah, 21589, Saudi Arabia. ${ }^{2}$ Departamento de Análisis Matemático, Facultad de Matemáticas, Universidad de Santiago de Compostela, Santiago de Compostela, 15782, Spain.

\section{Acknowledgements}

This project was funded by the Deanship of Scientific Research (DSR), King Abdulaziz University, under grant No. (88-130-35-HiCi). The authors, therefore, acknowledge technical and financial support of KAU.

Received: 24 November 2014 Accepted: 2 February 2015 Published online: 26 February 2015

\section{References}

1. Ebaid, A: Analysis of projectile motion in view of fractional calculus. Appl. Math. Model. 35, 1231-1239 (2011)

2. Kilbas, A, Srivastava, HM, Trujillo, JJ: Theory and Applications of Fractional Differential Equations. North-Holland Mathematics Studies, vol. 204. Elsevier, Amsterdam (2006)

3. Samko, SG, Kilbas, AA, Marichev, Ol: Fractional Integrals and Derivatives: Theory and Applications. Gordon \& Breach, Linghome (1993)

4. Podlubny, I: Fractional Differential Equations. Academic Press, San Diego (1999)

5. Baleanu, D, Guvenc, ZB, Machado, JAT: New Trends in Nanotechnology and Fractional Calculus Applications. Springer, New York (2010)

6. Baleanu, D, Golmankhaneh, AK, Nigmatullin, R, Golmankhaneh, AK: Fractional Newtonian mechanics. Cent. Eur. J. Phys. 8, 120-125 (2010)

7. Golmankhaneh, AK, Yengejeh, AM, Baleanu, D: On the fractional Hamilton and Lagrange mechanics. Int. J. Theor. Phys. 51, 2909-2916 (2012)

8. Rosales Garcia, JJ, Guia Calderon, M, Martinez Ortiz, J, Baleanu, D: Motion of a particle in a resisting medium using fractional calculus approach. Proc. Rom. Acad., Ser. A 14, $42-47$ (2013)

9. Rosales, JJ, Guia, M, Gomez, F, Aguilar, F, Martinez, J: Two dimensional fractional projectile motion in a resisting medium. Cent. Eur. J. Phys. 12, 517-520 (2014)

10. Belmekki, M, Nieto, JJ, Rodríguez-López, R: Existence of periodic solution for a nonlinear fractional differential equation. Bound. Value Probl. 2009, Article ID 324561 (2009)

11. Baleanu, D, Diethelm, K, Scalas, E, Trujillo, JJ: Fractional Calculus Models and Numerical Methods. Series on Complexity, Nonlinearity and Chaos. World Scientific, Boston (2012)

12. Priya, GS, Prakash, P, Nieto, JJ, Kayar, Z: Higher-order numerical scheme for fractional heat equation with Dirichlet and Neumann boundary conditions. Numer. Heat Transf., Part B, Fundam. 63, 540-559 (2013)

13. Yang, XJ: Advanced Local Fractional Calculus and Its Applications. World Science Publisher, New York (2012) 
14. Hayen, JC: Projectile motion in a resistant medium. Part 1: exact solution and properties. Int. J. Non-Linear Mech. 38, 357-369 (2003)

15. Hayen, JC: Projectile motion in a resistant medium. Part 2: approximate solution and estimates. Int. J. Non-Linear Mech. 38, 371-380 (2003)

Submit your manuscript to a SpringerOpen ${ }^{\circ}$ journal and benefit from:

- Convenient online submission

Rigorous peer review

- Immediate publication on acceptance

- Open access: articles freely available online

- High visibility within the field

- Retaining the copyright to your article

Submit your next manuscript at $\boldsymbol{s p r i n g e r o p e n . c o m ~}$ 